\title{
Studies of Catalyst-Controlled Regioselective Acetalization and Its Application to Single-Pot Synthesis of Differentially Protected Saccharides.
}

\author{
Jeonghyo Lee,,$^{\dagger}$ Sibin Wang, ${ }^{\dagger}$ Oleksii Zhelavskyi, ${ }^{\dagger}$ Alonso J. Argüelles, ${ }^{\dagger}$ Enoch Mensah, ${ }^{\dagger \$}$ Hao Guo, ${ }^{\dagger}$ \\ Rami Hourani, ${ }^{\dagger}$ Paul M. Zimmerman, ${ }^{\dagger *}$ and Pavel Nagorny. ${ }^{\dagger *}$
}

${ }^{\dagger}$ Chemistry Department, University of Michigan, 930 N. University Avenue, Ann Arbor, MI 48109

\begin{abstract}
Chiral Phosphoric Acid, Acetalization, Regioselective, Protection, Carbohydrates, Computational Studies, Catalysis on Solid Support
\end{abstract}

\begin{abstract}
This article describes the studies of regioselective acetal protection of monosaccharide-based diols using chiral phosphoric acids (CPAs) as the catalysts. These catalyst-controlled regioselective acetalizations were found to proceed with high regioselectivities (up to $>25: 1 \mathrm{rr}$ ) on various $D$-glucose, $D$-galactose, $D$-mannose and $L$-fucose derived 1,2-diols and could be carried with immobilized and recyclable catalyst. The utility of CPA-catalyzed acetalizations was further demonstrated for the regioselective single-pot preparation of various differentially protected mono- and disaccharide building blocks. The computational and mechanistic studies indicate complex temperature-dependent interplay of two reaction mechanisms, one involving an anomeric phosphate intermediate and another via concerted asynchronous formation of acetal. The computational models explain the steric factors responsible for the observed C2-selectivities and are consistent with the experimentally observed selectivity trends.
\end{abstract}

\section{INTRODUCTION}

Carbohydrates are essential molecules that not only serve as immediate energy sources, but are also associated with numerous biological activities. Accordingly, the studies of carbohydrates have been the focus of many ongoing investigation in the fields of organic chemistry, biochemistry, and drug discovery. ${ }^{1}$ However, in many instances, gaining access to complex oligosaccharides and glycoconjugates has been the bottleneck for the exploration of their biological and medicinal properties. ${ }^{2}$ Complex oligosaccharides are comprised of the simpler monosaccharides, and, not surprisingly, modern synthetic approaches to oligosaccharides strongly rely on the ability to access differentially protected building blocks. Synthesis of such differentially protected monosaccharides often requires multiple step sequences to differentiate numerous hydroxyl groups, and may suffer from low yields and tedious purifications due to the formation of undesirable regioisomers. ${ }^{3}$ To address the challenges associated with the selective functionalization of monosaccharides, numerous methods, including single pot telescoped protection approach by the Hung group, have been previously developed.4,5 However, the majority of such methods rely on the reagents and catalysts that discriminate between the axial and equatorial hydroxyl groups while only few methods exist for the selective differentiation of equatorial hydroxyl groups that are in similar steric and electronic environment ( $c f$. Scheme 1A).6,7 Pioneered by the Miller group (Scheme 1B), 8 the approaches based on the use of asymmetric catalysts to achieve site-selective functionalization of sugar-derived polyols have recently gained
Scheme 1. Selected examples of chiral catalyst-controlled differentiation of equatorial alcohols in carbohydrates

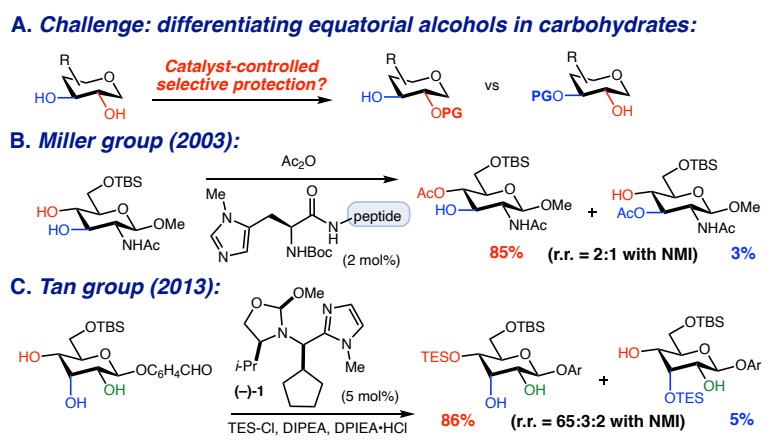

D. Nagorny group (2013):

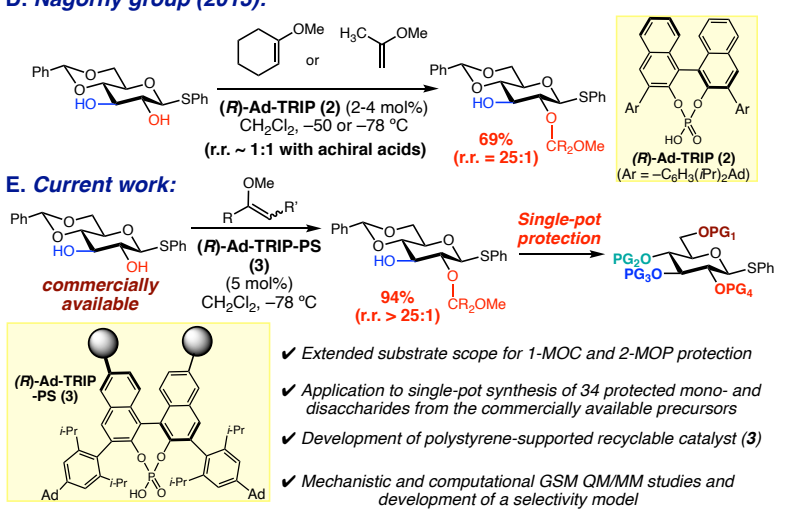


significant attention. ${ }^{8-11}$ While many efforts, most notoriously by the Kawabata, ${ }^{9}$ and Tang ${ }^{10}$ groups, have focused on exploring asymmetric catalysts for the selective acylation reactions, chiral catalyst-controlled site-selective phosphorylation, thiocarbonylation, sulfonation, silylation (Scheme 1C), acetalization and glycosylation have also been explored in the context of chiral catalyst-controlled functionalization of sugar-derived polyols. ${ }^{11}$

We have long-standing interests in utilizing chiral phosphoric acids as the catalysts leading to regio- and stereoselective acetal formation. ${ }^{12-14}$ As the part of these studies our group disclosed selective functionalization of monosaccharides using 1,1'-bi-2-naphthol (BINOL)-derived chiral phosphoric acids (CPAs) ${ }^{15}$ as the catalysts to direct regioselective acetalization of carbohydrate-derived 2,3-diols ( $c f$. Scheme 1D). ${ }^{13}$ This study demonstrated that $(R)$-CPA catalyst 2 could promote regioselective protection of $D$-glucose, $D$-galactose, $D$-mannose-derived diols and a $L$-fucose-derived triol with enol ethers such as dihydropyrane (DHP), 2-methoxypropene (2-MP), and 1-methoxycyclohexene (1-MOC). The reactions of $D$-glucose and $D$-galactose derivatives proceeded at the sites that cannot be directly functionalized with achiral reagent such as $n$-dibutyltin(IV) oxide. $6 \mathrm{~d}$ In addition, it was observed that the chirality of the catalyst played an important role as using the $(S)-2$ or achiral acids such $(\mathrm{PhO})_{2} \mathrm{PO}_{2} \mathrm{H}$ resulted in unselective reactions or selectivity switch.

Building on this approach, the current article expands the substrate scope and demonstrates the use of CPA-catalyzed regioselective acetalizations for single-pot synthesis ${ }^{16}$ of various differentially protected $D$-glucose, $D$-galactose and $D$-mannose mono- and disaccharides (Scheme 1E). These single-pot telescoped protocols proceed with high selectivities and efficiencies and require minimum purifications to access valuable protected building blocks from commercially available diol precursors. To address the challenges associated with high price of the catalyst 2 , we describe the development of immobilized catalyst $\mathbf{3}$ that was found to promote selective acetalization reactions often with higher selectivities and could be recycled and re-used. Finally, this article summarizes the mechanistic and computational QM/MM studies of the acetalization reaction mechanism and reveals that this transformation may proceed through an interplay of two different reaction mechanisms with the asynchronous concerted acetalization mechanism being dominant at low temperatures and leading to the observed selective formation of acetals. These insights into the reaction mechanism were used to develop a stereochemical model explaining the observed selectivity trends.

\section{DISCUSSION}

Selective protection of a single alcohol moiety as an acetal within a sugar-derived diol possessing alcohols in similar steric and electronic environment represents a challenge. Acid-catalyzed acetal formation is known to proceed through the intermediacy of highly reactive intermediates- oxocarbenium ions, which are known to react with nucleophiles indiscriminately. ${ }^{22 a}$ The resultant acetals are also acid labile, and equilibration/isomerization leading to the most thermodynamically stable product under the reaction conditions is not uncommon. Being very useful in organic synthesis and carbohydrate chemistry, mixed acetal protecting groups such as 1-methoxycyclohexyl (MOC) and 2methoxy-2-propyl (MOP) are very sensitive to acids and may easily hydrolyze and/or undergo the formation of 1,3dioxolanes and 1,3-dioxanes. ${ }^{17}$

Scheme 2. Site-selective MOC and MOP protection of monosaccharide-derived diols ${ }^{a}$

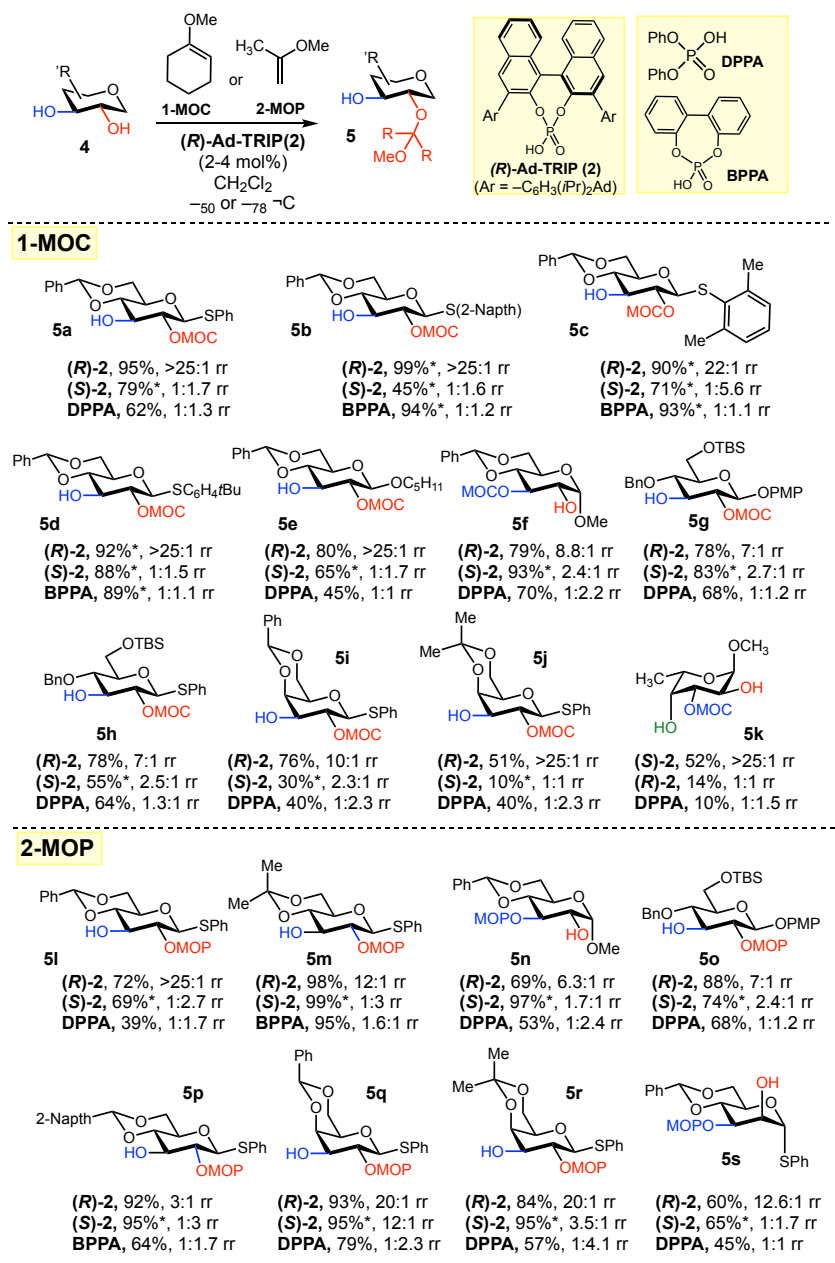

aThe reactions with 2-methoxypropene (2-MOP) were performed overnight at $-78{ }^{\circ} \mathrm{C}$ and the reactions with 1-methoxycyclohexene (1-MOC) were performed overnight at $-50 \stackrel{\circ}{\circ}$ on $0.056 \mathrm{mmol}$ scale $(0.042 \mathrm{M}$ solution) for $18-24 \mathrm{~h}$ in the presence of $4 \AA$ MS. The reaction with 4 a was performed on 2.78 mmol (1.0 g) scale. These rr values were determined by ${ }^{1} \mathrm{H}$ NMR analysis of the crude reaction mixtures of the products 5 and $\mathbf{6}$ as well as their acetylated derivatives. The values with asterisk* represent conversion determined by ${ }^{1} \mathrm{H}$ NMR analysis, and each entry represents an average of 2 experiments. 
With these considerations in mind, it was particularly surprising to observe that $(\boldsymbol{R})$-Ad-TRIP (2)-catalyzed formations of MOC and MOP acetals often proceeded with great levels of regiocontrol and in good yields ( $c f$. Scheme 2 and SI Scheme 1S).13,14 Remarkably, the control experiments with various achiral phosphoric acids such as DPPA and BPPA as well as $p$-TSA and CSA did not lead to selective reactions, and approximately equimolar mixtures of the $\mathrm{C} 2$ and $\mathrm{C} 3$ protected acetals were obtained in each instance in diminished yields. Similarly, the use of enantiomeric (S)Ad-TRIP catalyst 2 often resulted in diminished yields and selectivities, and in the instance of $\mathbf{5 c}, \mathbf{5 l}, \mathbf{5 m}$, and $\mathbf{5 p}$ the selectivity was reversed to produce significant amounts of the C3-regioisomer. Not surprisingly, the use of $L$ - rather than $D$-sugars such as $L$-fucose-derived triol $\mathbf{4 k}$ required $(S)$ - rather than $(R)-2$ to achieve selective protection of the C3 position.

The $D$-glucose derivatives possessing 4,6-benzylidene acetal protection and featuring $\beta$-configuration at the anomeric position exhibited highest levels of regiocontrol exclusively providing the $\mathrm{C} 2$-protected products $\mathbf{5 a}-\mathbf{5 e}$ and 51. The size and nature of the anomeric substituent with $\beta$ configuration seemed to have little effect on the reaction selectivity; however, the changes in the 4,6-acetal moiety seemed to have a significant effect. Thus, the (R)-2-catalyzed 2-MOP protection leading to 2-naphthyl product $\mathbf{5 p}$ proceed with the significantly lower C2-selectivity (3:1 rr) then for the corresponding benzylidene acetal derivative $\mathbf{5 l}$ ( $>25: 1 \mathrm{rr}$ ). In line with this observation, the analogous to $\mathbf{5 l}$ acetonide derivative $\mathbf{5 m}$ was produced in only $12: 1 \mathrm{rr}$ while the MOP-derivative 5o in 7:1 rr. Similarly, MOC protection leading to the derivatives $\mathbf{5 g}$ and $\mathbf{5 h}$ lacking the 4,6-benzylidene acetal moiety were produced with lower $\mathrm{C} 2$ selectivities (5:1 and 7:1 rr, correspondingly) than for the benzylidene acetal containing products $\mathbf{5 a - 5 e}$.

Finally, it should be noted that the presence of the axial substitution next to the C2/C3 diol seems to direct the protection to the position furthest away from the axial substitution. Thus, both the MOC and 2-MOP protection of $D$-galactose derivatives with $\beta$-anomeric configuration, $\mathbf{4 i}$ and $\mathbf{4 j}$, provided C2-protected products $\mathbf{5 i}, \mathbf{5 j}, \mathbf{5 q}$ and $\mathbf{5 r}$ in good-to-excellent selectivities and yields. Following this trend, $D$-glucose derivative with $\alpha$-methoxy configuration $\mathbf{4 f}$ provided the $\mathrm{C} 3$ (rather than the $\mathrm{C} 2$ ) products $\mathbf{5 f}$ and $\mathbf{5 n}$ in moderately good selectivities (8.8:1 rr and 6.3:1 rr, correspondingly).

With these results in hand, our subsequent studies were focused on demonstrating that regioselective products $\mathbf{5 a -}$ 5s are of great utility for the preparation of differentially protected monosaccharide derivatives from commercially available building blocks such as $\mathbf{4 a}$. Inspired by the streamline method for the single-pot synthesis of various $D$ glucose derivatives by the Hung and coworkers, ${ }^{4}$ our subsequent studies focused on the derivatization of $\mathbf{5 a}$ ( $c f$.
Scheme 3). Considering that both the MOC and 2-MOP protecting groups are stable to a variety of basic conditions, but highly labile in the presence of an acid, our single pot protocol included in situ removal of the MOC protection with 0.05 $\mathrm{M} \mathrm{HCl}$ without deprotecting the 4,6-benzylidine acetal (cf. Scheme 3) ${ }^{17}$ The high volatility of $0.05 \mathrm{M} \mathrm{HCl}$ solution in DCM was also suitable for the overall single-pot functionalization process as it could be removed by passing a nitrogen stream over the reaction mixture without the necessity of carrying the reaction work up.

Scheme 3. Regioselective single-pot synthesis of C2/C3 protected monosaccharide derivatives enabled by $(R)$ Ad-TRIP(2)-catalyzed MOC-protection ${ }^{\mathrm{a}}$

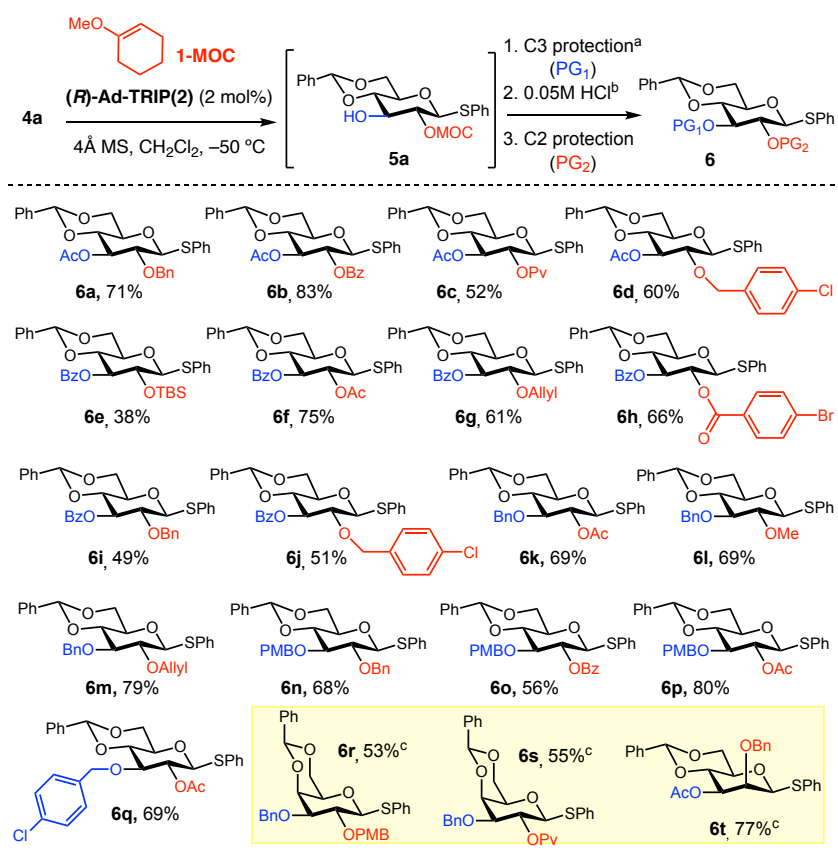

aIsolated yield after one-pot sequence leading to 6 . The reaction mixtures were concentrated by the stream of $\mathrm{N}_{2}$ prior to each protection step. Unless noted otherwise, these experiments were carried on $0.056 \mathrm{mmol}$ scale. MOC protection was accomplished as described in Scheme 2. aAcylations were carried using $\mathrm{Ac}_{2} \mathrm{O}$ (1.1 equiv), DMAP (10 mol\%), $\mathrm{Et}_{3} \mathrm{~N}$ (5.0 equiv) or $\mathrm{BzCl}$ (1.2 equiv), DMAP (20 mol\%), Py (10 equiv). Alkylation was accomplished by addition of $\mathrm{NaH}$ (1.2 equiv), alkyl halide (1.1 equiv), TBAI ( 0.1 equiv) and DMF (cat); bThe MOC cleavage was achieved by addition of $0.05 \mathrm{M} \mathrm{HCl}$ in $\mathrm{CH}_{2} \mathrm{Cl}_{2}(\mathrm{pH}=5)$. Please refer to SI for the detailed description of these single-pot protocols; cCompounds 6r-6t were generated using MOPprotected intermediates $\mathbf{5 q}$ and $\mathbf{5 s}$.

The one-pot transformations depicted in Scheme 3 commenced with CPA (2)-directed regioselective acetalization, and the resultant C2-acetal $\mathbf{5 a}$ was successfully obtained in full conversion. At this point, the mixture was dried by sequentially applying a gentle $\mathrm{N}_{2}$ flow and flask evacuation before the next step. Initially the crude mixture containing 
compound 5a was subjected to free C3-hydroxyl group protection without affecting the $\mathrm{C} 2$-acetal. Since mixed acetals are acid- and heat- labile, acidic conditions and high reaction temperatures were avoided. The regioselective acylations of the $\mathrm{C} 3-\mathrm{OH}$ were accomplished using (RCO) $)_{2} \mathrm{O} / \mathrm{Py}$ or $\mathrm{RCOCl} / \mathrm{Et}_{3} \mathrm{~N}$ in the presence of catalytic DMAP (substrates 6a-6j). Alternatively, the C3-alkylation was accomplished using alkyl halides and sodium hydride as the base in the presence of catalytic TBAI (6k-6t). The C2 MOC acetal was removed by adding excess of $\mathrm{HCl}$ solution in DCM to the reaction mixture. The resultant acidic solutions were concentrated by passing $\mathrm{N}_{2}$ stream to blow out the volatiles and subsequent evacuation of the reaction vessel, and the concentrated crude oils were subjected to the $\mathrm{C} 2$-protection to provide products 6a-6t in good yields $49-83 \%$ overall yield). In addition to the $D$-glucose-based derivatives $\mathbf{6 a - 6 q}$, this method was also successfully applied to generate $D$-galactose-based substrates $\mathbf{6 r}$ and $\mathbf{6 s}$ as well as $D$-mannosederived substrate $\mathbf{6 t}$ using MOP (rather than MOC) protection strategy. It is noteworthy that $D$-mannose derivative $\mathbf{5 s}$ contained MOP acetal at the $\mathrm{C} 3$ position, which required functionalization in a reverse way (C2-benzylation, C3-acetal cleavage, and C3-acetylation) to afford compound $\mathbf{6 t}$ in good overall yield $(77 \%)$ and selectivity $(>12: 1 \mathrm{rr}$ for the first step).

It is noteworthy that while some of the derivatives from 6k-6q might be potentially accessible through one-pot glucose functionalization strategy developed by the Hung group, one-pot synthesis of derivatives 6a-6j featuring the C3 acyl protection has not been previously described and would be challenging to accomplish via a single-pot protocol in good yields. Similarly, previously published one-pot protection protocols could not be readily adopted to the synthesis of $D$-galactose and $D$-mannose derivatives similar to 6r-6t. Finally, the operational simplicity for the deprotection of the MOC and MOP groups allows to expand the scope of the protecting groups (to include substrates like 6e) and achieve flexibility in controlling the $\mathrm{C} 2 / \mathrm{C} 3$ protection to generate regioisomeric substrates like $\mathbf{6 d}$ and $\mathbf{6 q}$ in similar yields ( $60 \%$ and $69 \%$, correspondingly).

Scheme 4. Regioselective single-pot synthesis of differentially protected $D$-glucose derivative from $4 \mathrm{a}^{\mathrm{a}}$

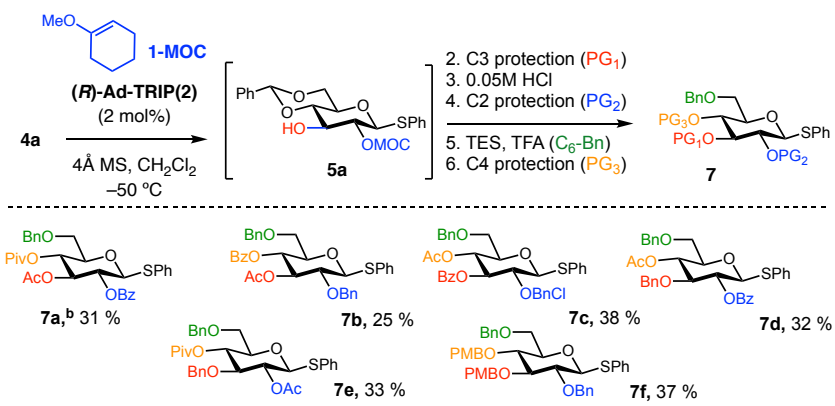

aIsolated yield after one-pot sequence leading to 7. The reaction mixtures were concentrated by the stream of $\mathrm{N}_{2}$ prior to each protection step. MOC protection was accomplished as described in Scheme 2. Unless noted otherwise, these experiments were carried on $0.056 \mathrm{mmol}$ scale. Acylations were carried using $\mathrm{Ac}_{2} \mathrm{O}$ (1.1 equiv), DMAP (10 mol\%), $\mathrm{Et}_{3} \mathrm{~N}$ (5.0 equiv) or BzCl (1.1 equiv), DMAP (10 mol\%), Et ${ }_{3} \mathrm{~N}$ (5.0 equiv). Alkylation was accomplished by addition of $\mathrm{NaH}$ (1.2 equiv), alkyl halide (1.1 equiv), TBAI ( 0.1 equiv) and DMF (cat); benzylidene acetal cleavage was accomplished using $\mathrm{Et}_{3} \mathrm{SiH}$ (5 equiv), $\mathrm{CF}_{3} \mathrm{CO}_{2} \mathrm{H}$ (5 equiv), DCM, $0 \stackrel{\circ}{\circ}, 2 \mathrm{~h}$. bThe synthesis of $7 \mathrm{a}$ was carried on $1.0 \mathrm{~g}$ scale. Please refer to SI for the detailed description of these single-pot protocols.

These transformations could be further combined with benzylidene acetal opening/C4 protection steps to accomplish single-pot generation of differentially protected $D$-glucose derivatives 7a-7f depicted in Scheme 4. Thus, the previously developed protocol included regioselective benzylidene acetal reduction with $\mathrm{CF}_{3} \mathrm{CO}_{2} \mathrm{H} / \mathrm{Et}_{3} \mathrm{SiH}$ leading to product containing free $\mathrm{C} 4$ alcohol that was subsequently protected. These single-pot transformations proceeded in 25$38 \%$ overall yield ( $\sim 85 \%$ yield per operation) with high levels of overall regiocontrol. These procedures were scalable, and the derivative $\mathbf{7 a}$ was made on $1.0 \mathrm{~g}$ scale ( $31 \%$ yield).

Scheme 5. Regioselective single-pot protection and subsequent glycosylation of $D$-glucose and $D$-mannose derivatives $4 a$ and $4 s^{a}$

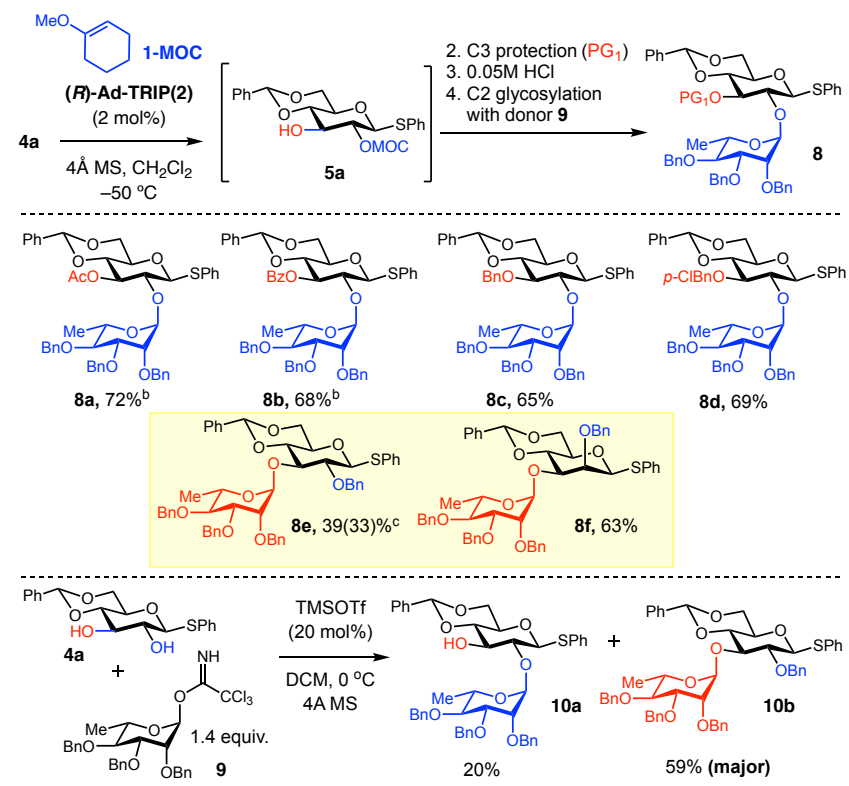

assolated yield after one-pot sequence leading to $\mathbf{8}$. The C3 protection/C2 deprotection sequence was accomplished as described in Scheme 3, and the resultant mixture was azeotropically dried prior to the glycosylation step. Unless noted otherwise, these experiments were carried on $0.056 \mathrm{mmol}$ scale. bThe final glycosylation step to form $\mathbf{8 a}$ and $\mathbf{8 b}$ was accomplished with 2.5 equiv of 9 and TMSOTf ( $20 \mathrm{~mol} \%$ ) in DCM at 0 o $C$ in the presence of $4 \AA$ MS. The glycosylations leading to $8 \mathrm{c}$ $8 f$ was accomplished in the presence of 1.4 equiv of 9. The resultant product contained inseparable impurity and was $85 \%$ 
pure by ${ }^{1} \mathrm{H}$ NMR analysis. The yield in parenthesis represents corrected for the impurity yield.

The aforementioned regioselective manipulations of $\mathbf{4 a}$ could be combined with glycosylation to accomplish concomitant protection and glycosylation of the $\mathrm{C} 2$ and $\mathrm{C} 3$ positions ( $c f$. Scheme 5). Thus, CPA-catalyzed C2-protection with MOC was followed with acylation (8a and $\mathbf{8 b}$ ) or alkylation (8c or $\mathbf{8 d}$ ), subsequent MOC group cleavage, and glycosylation with $L$-rhamnose trichloroacetimidate 9 to provide derivatives 8a-8d in good yields (65-72\%) as single regioisomers with the glycosylated $\mathrm{C} 2$ position. This protocol was also extended to other sugars such as $D$-mannose (4s) to provide the protected and glycosylated derivative $\mathbf{8 f}$ as the single regioisomer in $63 \%$ yield. The inclusion of the additional C2 protection/C3 deprotection sequence also allowed to achieve the reverse glycosylation of the $\mathrm{C} 3$ position and resulted in the derivative $\mathbf{8 e}$ in moderate yield (33\%). Importantly, the C3 hydroxyl group in $4 a$ is more reactive in glycosylation with $\mathbf{9}$, and the direct glycosylation of 4a provides $\sim 3: 1$ mixture of the regioisomeric products 10b and 10a in 79\% overall yield. Correspondingly, the described strategy offers significant advantages for the synthesis of C2 glycosides such as $\mathbf{8 a - 8 d}$.

Scheme 6. Regioselective single-pot protection and subsequent C1 glycosylation with $D$-glucose derivatives $4 a^{a}$
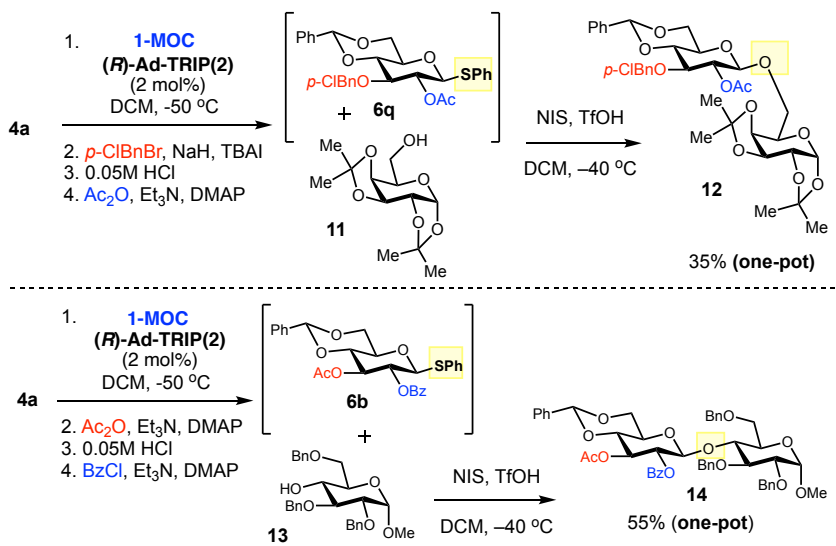

aIsolated yield after one-pot sequence leading to $\mathbf{8}$. The C3 protection/C2 deprotection sequence was accomplished as described in Scheme 3, and the resultant mixture was azeotropically dried prior to the glycosylation step. Unless noted otherwise, these experiments were carried on $0.056 \mathrm{mmol}$ scale. bThe final glycosylation step to form $\mathbf{8 a}$ and $\mathbf{8 b}$ was accomplished with 1.5 equiv of $\mathbf{6 b}, 1.0$ equiv of $\mathbf{1 1}$ or $\mathbf{1 3}$, and TMSOTf (20 mol\%) and NIS (2.0 equiv) in DCM at $0{ }^{\circ} \mathrm{C}$ in the presence of 4 Å MS.

The substrates $\mathbf{6 a - 6 t}$ carry a thiophenol moiety at the anomeric position, and, hence, could serve as glycosyl donors ( $c f$. Scheme 6). Thus, we selected two derivatives of $\mathbf{4 a}$ (i.e. $\mathbf{6 b}$ and $\mathbf{6 q}$ ) and demonstrated that the regioselective protection leading to $\mathbf{6} \mathbf{b}$ and $\mathbf{6 q}$ could be combined with glycosylation using acceptors $\mathbf{1 1}$ and $\mathbf{1 3}$ and leading to single pot formation of disaccharides $\mathbf{1 2}$ and $\mathbf{1 4}$. While the yield of 12 (35\%) was diminished due to the lability of acetonide moieties in $\mathbf{1 1}$ and $\mathbf{1 2}$ under the acidic conditions, the single pot synthesis of $\mathbf{1 4}$ proceeded in good yield (55\%) and selectivity.

We believe that the aforementioned results demonstrate great potential of CPA-catalyzed regioselective acetalizations are of great use for the single pot protection and glycosylation of monosaccharides. While this method allows to expand the repertoire of sugar derivatives and transformations developed from Hung, it suffers from the obligatory use of expensive chiral acid (R)-Ad-TRIP (2). However, these costs could be significantly reduced if the catalyst is immobilized on solid support as numerous recent studies suggest that such catalysts could be recovered and recycled or employed for the catalysis in continuous flow. ${ }^{18}$

Scheme 7. Synthesis and applications of polystyrenesupported CPA catalyst $(R)$-Ad-TRIP-PS (3)

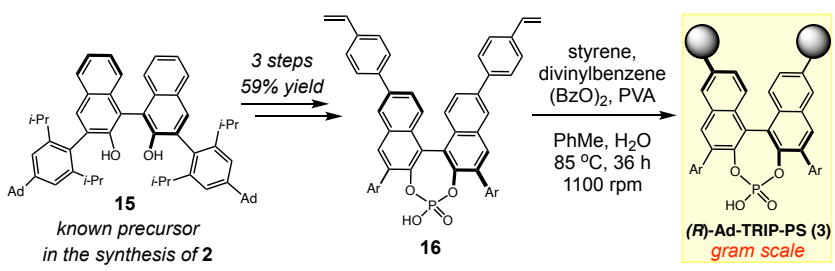

Therefore, our subsequent efforts were focused on developing a catalytically active immobilized version of $\mathbf{2}$ (cf. Scheme 7 and Scheme 3S-SI). ${ }^{19}$ Based on the previously developed strategies for TRIP immobilization, ${ }^{20}$ we investigated this variant for the synthesis of immobilized on polystyrene support catalyst (R)-Ad-TRIP-PS (3). While the previously published route did not lead to catalytically active in acetalization reactions (R)-Ad-TRIP-PS (3), we were able to modify this approach (cf. Scheme 7 ) to produce a viable immobilized catalyst.

Scheme 8. Regioselective 2-MOP protection with immobilized catalyst $(R)$-Ad-TRIP-PS (3)

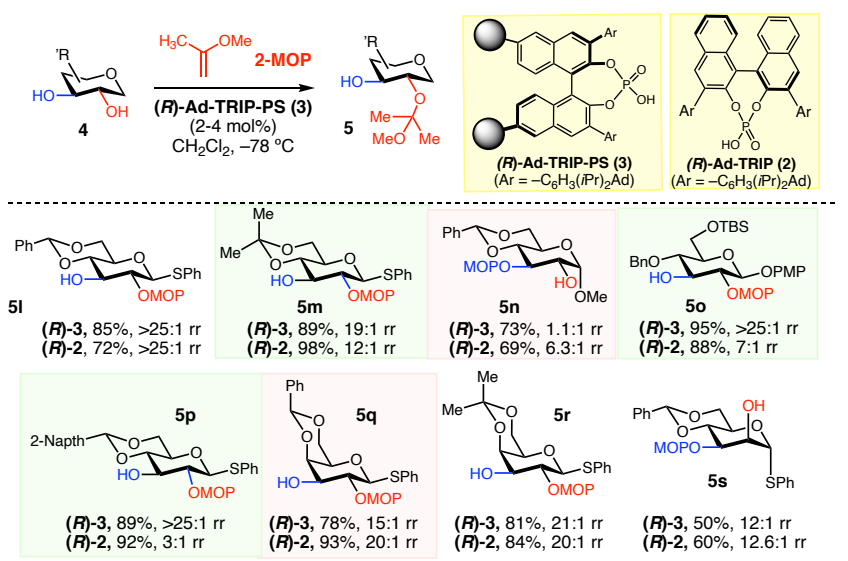


aThe reactions were performed overnight at $-78^{\circ} \mathrm{C}$ on 0.056 mmol scale $(0.042 \mathrm{M}$ solution) for $18-24 \mathrm{~h}$ in the presence of $4 \AA$ MS. These rr values were determined by ${ }^{1} \mathrm{H}$ NMR analysis of the crude reaction mixtures of the products $\mathbf{5}$ as well as their acetylated derivatives. Each entry represents an average of 2 experiments.

With the successful synthesis of gram quantities of $\mathbf{3}$ in hand, the use of this catalyst for the generation of 2-MOP protected derivatives 5l-5s was investigated ( $c f$. Scheme 8). In three instances (5l, $\mathbf{5 r}$ and $\mathbf{5 s}$ ), the results obtained with the immobilized catalyst $\mathbf{3}$ matched the results previously obtained for CPA 2 (cf. Scheme 2).

Scheme 9. Single-pot regioselective protection of $(D)$ glucose derivative $4 a$ with immobilized catalyst $(R)$-AdTRIP-PS (3) ${ }^{a}$

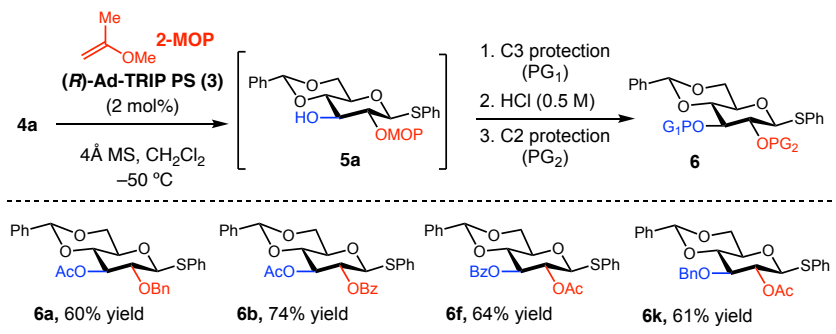

aThe reaction mixtures were concentrated by the stream of $\mathrm{N}_{2}$ prior to each protection step. Unless noted otherwise, these experiments were carried on $0.05 \mathrm{mmol}$ scale. MOP protection was accomplished as described in Scheme 8. aAcylations were carried using $\mathrm{Ac}_{2} \mathrm{O}$ (1.1 equiv), DMAP (10 mol\%), $\mathrm{Et}_{3} \mathrm{~N}$ (5.0 equiv) or $\mathrm{BzCl}$ (1.2 equiv), DMAP (20 mol\%), Py (10 equiv). Benzylation was accomplished by addition of $\mathrm{NaH}$ (1.2 equiv), BnBr (1.1 equiv), TBAI (0.1 equiv) and DMF (cat) The yield represents an average of 2 runs.

Remarkably, in three instances (5m, 5o, and 5p), CPA 3 provided significantly higher regioselectivities than acid 2 (25:1 rr vs 3:1 rr for $\mathbf{5 p}$, 25:1 rr vs. 6:1 rr for 5o, and 19:1 rr vs 12:1 rr for $\mathbf{5 m}$ ). In addition, in two instances (5n and $\mathbf{5 q}$ ), the selectivities obtained with 3 were lower than the selectivities obtained with 2 (1:1 rr vs 6:1 rr for $\mathbf{5 n}$ and 20:1 rr vs 15:1 rr for $\mathbf{5 q}$ ). These observations suggest that the additional structural features in $\mathbf{3}$ that are not present in $\mathbf{2}$ and include the linker and polystyrene matrix may impact the steric interactions between the CPA and substrate, which could have both positive or negative consequences for the reaction outcome. Importantly, in all of these studies immobilized catalyst $\mathbf{3}$ could be conveniently separated away from $4 \AA$ MS, recovered, and, if necessary, regenerated and recycled ( $c f$. Table $1 \mathrm{~S}-\mathrm{SI}$ ).

In analogy to the results in Schemes 3-5, the regioselective MOP protection with 3 could be used to accomplish one-pot formation of differentially protected at the C2/C3 positions derivatives $\mathbf{6 a}, \mathbf{6 b}, \mathbf{6 f}, \mathbf{6 k}$ ( $c f$. Scheme 9), and the yields and selectivities in these cases are similar to what was observed in the studies described in Scheme 3. In all of these cases, catalyst $\mathbf{3}$ was conveniently removed by filtration and recycled after the formation of MOP derivative $\mathbf{5 a}$.

Altogether, these studies demonstrate the utility of CPAcatalysts for the regioselective preparation of differentially protected monosaccharide derivatives and help to expand single-pot methods previously developed by Hung and others. ${ }^{4,5}$ In addition, the development and application of immobilized catalyst $\mathbf{3}$ allows to achieve catalyst recyclability and make these transformations potentially amenable to continuous flow. However, in order to further expand the substrate scope and develop a predictive model for the regioselective protection with 2-MOP and MOC groups is highly desired. Therefore, our subsequent studies were focused on experimental and computational exploration of the mechanism for the regioselective acetalizations described in Scheme 2. Both MOC and MOP acetalizations demonstrated unusual selectivity versus temperature profiles ( $c f$. Scheme 10). Thus, both types of acetalization were unselective at the temperatures above $-30 \stackrel{\circ}{\circ}$, but demonstrated significant increase in selectivity once cooled below $-50 \stackrel{\circ}{ } \mathrm{C}$. This suggests that the acetalization mechanism prevailing at the temperatures below $-50 \stackrel{\circ}{ } \mathrm{C}$ might be different from the reaction mechanism at higher temperatures.

\section{Scheme 10. Selectivity vs. temperature profile for the MOC and 2-MOP protection of $4 \mathrm{a}^{\mathrm{a}}$}
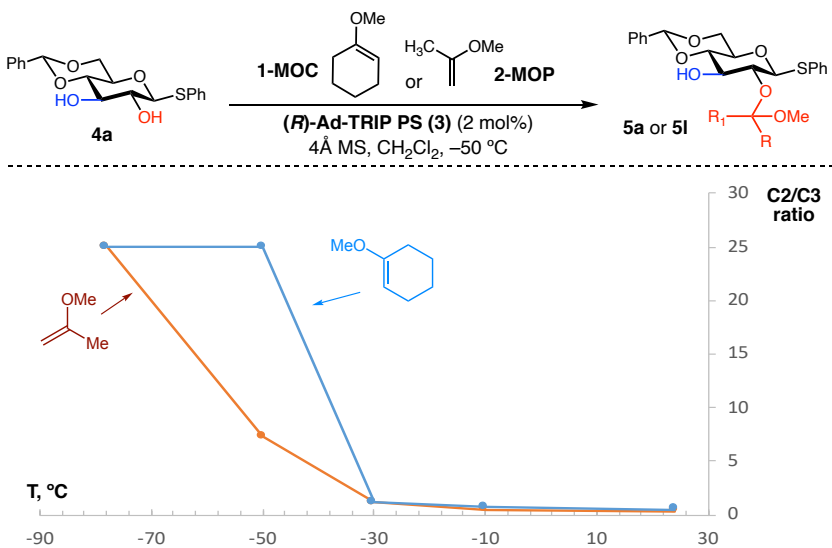

aThe reactions with 2-methoxy-2-propene (2-MOP) and 1methoxy-1-cyclohexene (1-MOC) were performed overnight at the specified temperature on $0.056 \mathrm{mmol}$ scale $(0.042 \mathrm{M}$ solution) in the presence of $4 \AA \mathrm{AS}$. The $\mathrm{C} 2 / \mathrm{C} 3$ ratio was determined by ${ }^{1} \mathrm{H}$ NMR analysis of the crude mixture.

With these observations in mind, three different reaction mechanisms depicted in Scheme 11 were proposed. These involved the conventional mechanism proceeding through the oxocarbenium ion (option A), ${ }^{21,22}$ as well as direct synchronous or asynchronous concerted addition, as previously proposed by us for the CPA-catalyzed spiroketalizations (option B). ${ }^{12 c}$ Alternatively, the catalyst may react with the MOC or 2-MOP enol ethers to form a phosphate intermediate, which then proceeds through $\mathrm{S}_{\mathrm{N}} 1$ or $\mathrm{S}_{\mathrm{N}} \mathrm{i}$ mecha- 
nisms that were observed by us for the CPA-catalyzed glycosylation of 6-dEB (option C). ${ }^{12 b, 23}$ Differentiating these mechanistic options computationally represents a challenging task as it requires finding and optimizing conformationally flexible transition state structures. To address some of these challenges, our computational studies used the reaction-path and transition-state search tool called the Growing String Method (GSM).24,25 The large size of the system (207 atoms) motivated a Quantum Mechanics/Molecular Mechanics (QM/MM) approach to make the reaction path analysis feasible. The QM/MM borders were set on the C-C bond at the 3,3'-positions of the chiral catalyst (R)-2 placing bulky hydrocarbon moieties in the MM region ( $c f$. Scheme 7S - SI).

\section{Scheme 11. Postulated acetalization mechanisms}

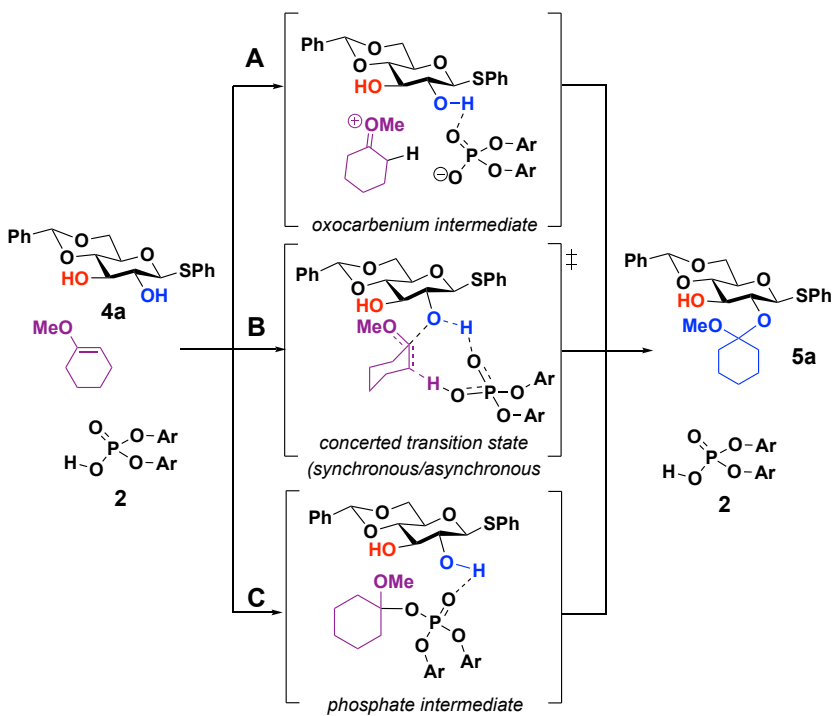

Reaction pathways were determined using GSM for the 20 lowest energy conformers for each path (C2 and C3 in each mechanism, $c f$. SI). The concerted mechanism was investigated first, beginning from the complex involving the substrate H-bonded to $(\boldsymbol{R})$-2 (Figure 1A). The reaction pathway shows an asynchronous concerted transformation: the CPA protonates the enol ether (PG) to generate an oxocarbenium cation that then reacts with the $\mathrm{OH}$-group of the sugar substrate 4a ( $\mathrm{TS}_{1}$ for C2-path and $\mathrm{TS}_{2}$ for C3-path). The resultant CPA anion deprotonates the OH-group synchronously with oxygen nucleophilic attack on the oxocarbenium cation. The lowest energy activation barriers among the 20 conformers of each TS favour C2-protected product $5 \mathbf{a}$ formation at $223 \mathrm{~K}\left(\Delta \Delta \mathrm{G}_{223}^{\ddagger}=-1.2 \mathrm{kcal} / \mathrm{mol}\right)$ and 273K $\left(\Delta \Delta \mathrm{G}_{273}^{\ddagger}=-1.0 \mathrm{kcal} / \mathrm{mol}\right)$ (Table 2). Activation energies $\left(\Delta G^{\ddagger}, \mathrm{kcal} / \mathrm{mol}\right)$ for this mechanism $(21.1$ and 22.3 $\mathrm{kcal} / \mathrm{mol}$ for C2- and C3-pathways respectively) are slightly higher than expected from the experimental data (18-19 $\mathrm{kcal} / \mathrm{mol}$ for an overnight reaction at $-50{ }^{\circ} \mathrm{C}$ ). This concerted mechanism, which does not show an oxocarbenium intermediate (Scheme 11, Path A), does bear similarities with the oxocarbenium mechanism. In particular, the oxocarbenium ion appears along the reaction path at the transition state structure, which identifies the concerted mechanism as asynchronous (Scheme 11, Path B).

The two-elementary-step phosphate-mediated mechanism (Scheme 11, Path C) was investigated next. To do so, 20 conformationally distinct reaction pathways involving (R)-(2) and 1-methoxycyclohexene (PG) were examined. The phosphate intermediate is reached via asynchronous protonation of the enol ether PG followed by nucleophilic attack with the same phosphoric acid oxygen that participated in substrate protonation. The activation energy for the lowest barrier phosphate formation step is 14.7 $\mathrm{kcal} / \mathrm{mol}$. Unlike our previous studies of the CPA-catalyzed glycosylations, the covalent phosphate intermediate formation was not observed by low temperature ${ }^{31} \mathrm{P},{ }^{13} \mathrm{C}$ or ${ }^{1} \mathrm{H}$ NMR studies. This is not surprising, however, as the computational studies indicate that the covalent phosphate intermediate is $4.4 \mathrm{kcal} / \mathrm{mol}$ less stable than the reactant complex. Keeping in mind the low energy barrier for its formation and dissociation back, this step should be considered as being in equilibrium. During the reaction, the phosphate acetal would be formed only in trace quantities and exist as a steady-state intermediate.

The second step of the phosphate-mediate pathway (Path C) has a reaction energy profile more consistent with a concerted $S_{\mathrm{N}} \mathrm{i}$ mechanism rather than an $\mathrm{S}_{\mathrm{N}} 1$ mechanism, as no stable carbocation intermediates were detected ( $c f$. Figure 1A). In this step the OH-group of 2,3-diol 4a attacks the carbon atom of the methoxy cyclohexane fragment of the phosphate intermediate at the same time as the C-O phosphate bond cleaves. This second step is rate-limiting and shows the opposite regioselectivity compared to the concerted mechanism. That is, at both temperatures of interest the C3protection was favored over the C2-protection $\left(\Delta \Delta \mathrm{G}_{223}^{\ddagger}=1.5\right.$ $\mathrm{kcal} / \mathrm{mol}$ and $\Delta \Delta \mathrm{G}_{273}^{\ddagger}=-1.6 \mathrm{kcal} / \mathrm{mol}$ ). Surprisingly, the activation energies of the rate-limiting step were also close to the ones computed for the concerted mechanism (Figure 1A).

The energy diagram summarizing our computational studies is provided in Figure 1A. Even though there was a slight preference for the phosphate mechanism, an obvious assignment of operating mechanism is not possible due to the energy difference being within the errors of the model. This situation, however, allows us to postulate that the concerted mechanism is preferred at low $\mathrm{T}$, and the phosphate mechanism at high T. Invoking this switch in mechanism comes with a change in regioselectivity that nicely explains the experimental results depicted in Scheme 10. At low temperatures, the concerted mechanism (Path B) would therefore give rise to high C2-selectvity $\left(\Delta \Delta \mathrm{G}^{\ddagger}(\mathrm{C} 2-\mathrm{C} 3)=-1.2\right.$ $\mathrm{kcal} / \mathrm{mol}$ ). At higher temperatures the phosphate mechanism (Path C) likely results in increased amounts of the C3 acetalization product $\left(\Delta \Delta \mathrm{G}^{\ddagger}(\mathrm{C} 2-\mathrm{C} 3)=1.5 \mathrm{kcal} / \mathrm{mol}\right)$.

With the mechanistic picture in hand, transition state 
Figure 1. Computational exploration of the reaction mechanism and stereochemical model for the regioselectivity

A. Energy diagram depicting potential reaction mechanisms at $223 \mathrm{~K}$

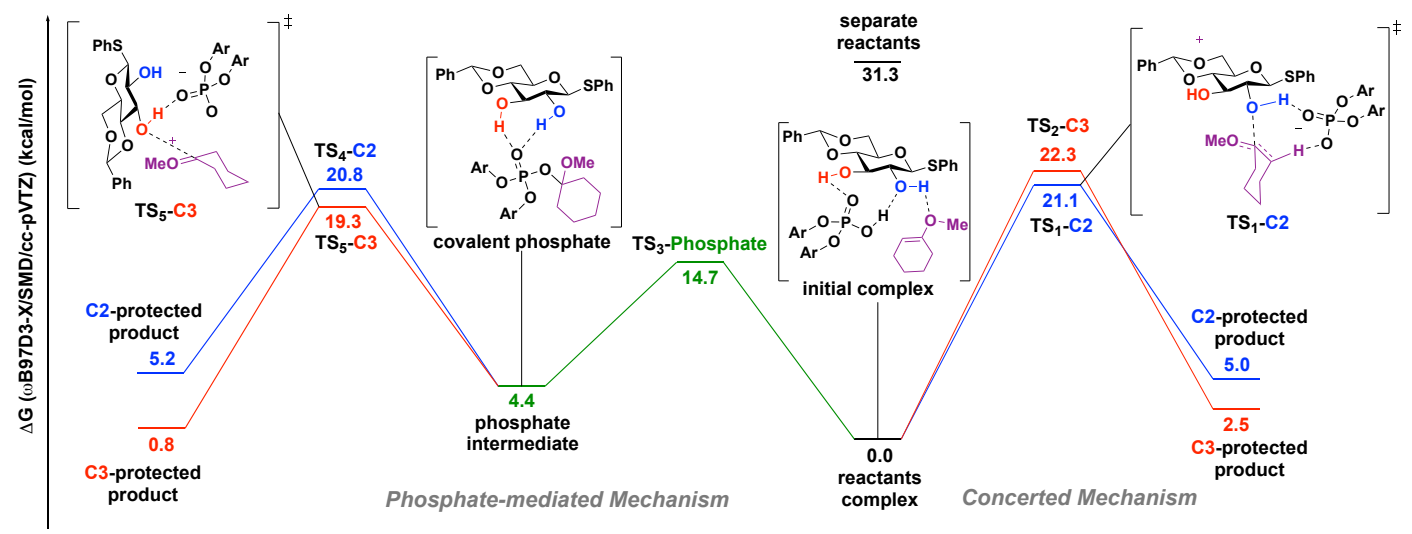

B. Noncovalent interaction analysis for regiocontrolling transition states $\mathrm{TS}_{1}$ and $\mathrm{TS}_{2}$ for concerted mechanism at $223 \mathrm{~K}$
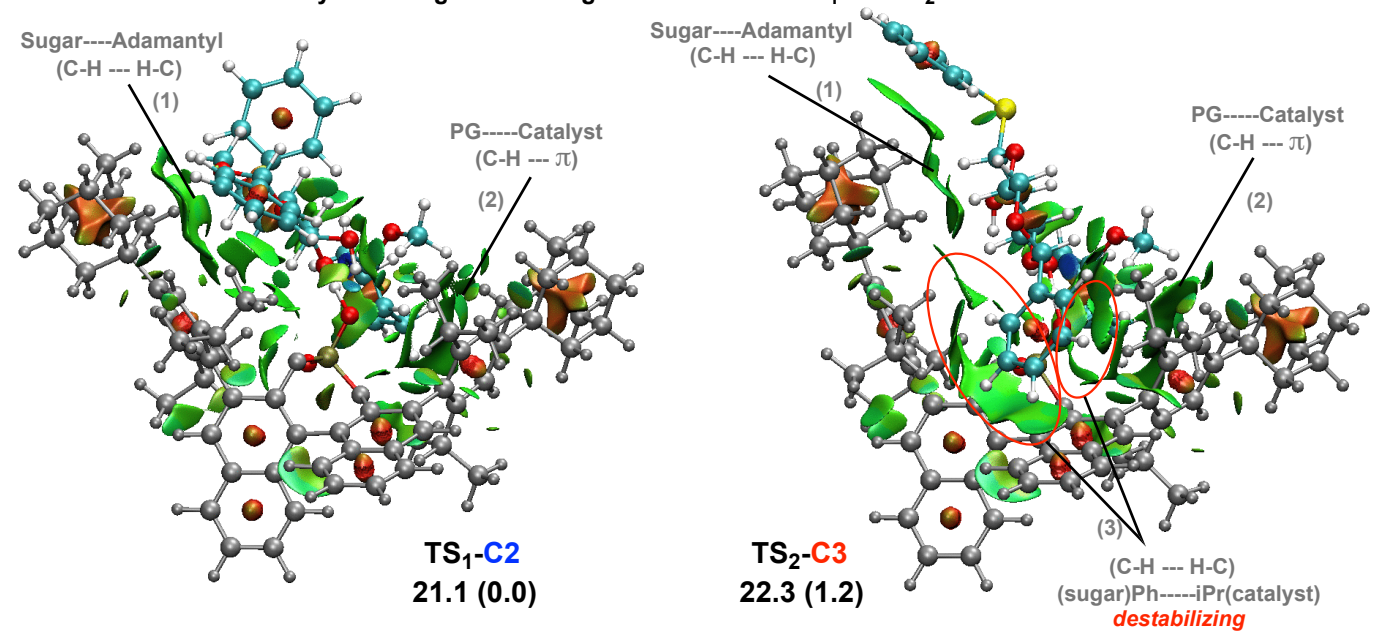

C. Noncovalent interaction analysis for regiocontrolling transition states $\mathrm{TS}_{4}$ and $\mathrm{TS}_{5}$ for phosphate-mediated mechanism at $223 \mathrm{~K}$
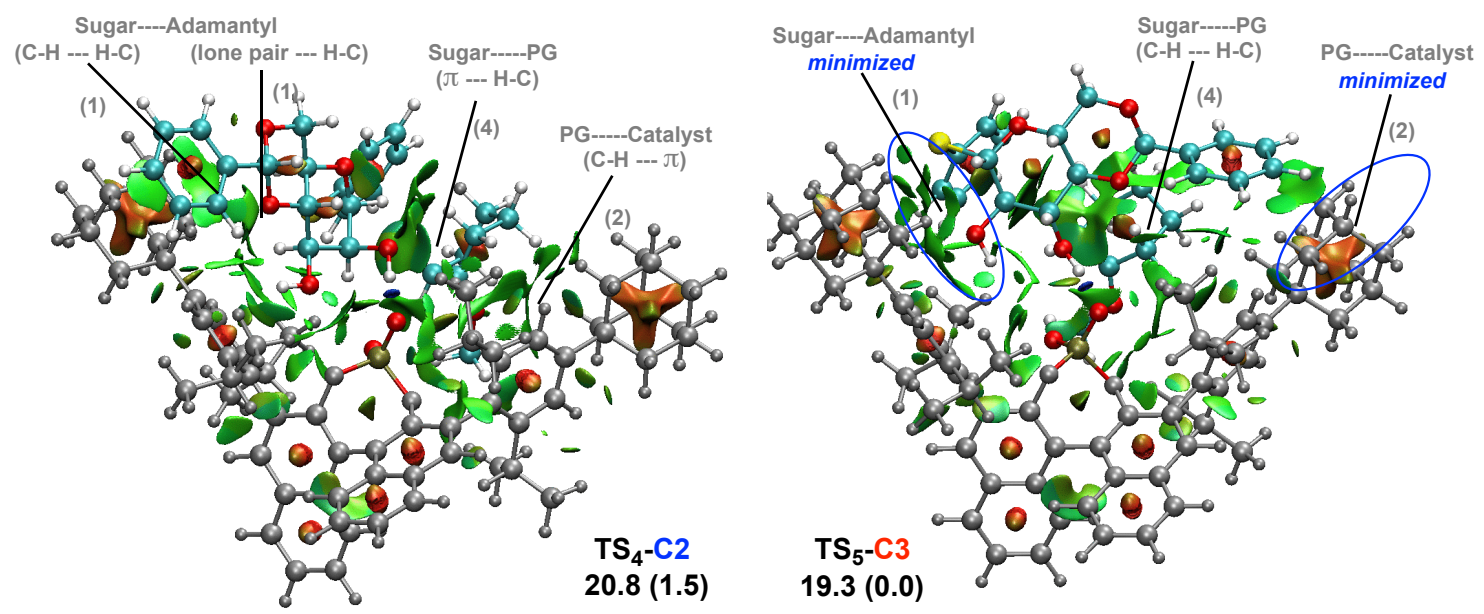
analysis was performed to identify the key structural features leading to different selectivities in each mechanism. The non-covalent interactions (NCIs) are known to be crucial in stabilizing or destabilizing the transition states when changes in energy by $1-2 \mathrm{kcal} / \mathrm{mol}$ can dramatically change the reaction selectivity. The graphical analysis ${ }^{26}$ delineates NCI into three categories: blue, strong attraction; green, weak interaction; and red, strong repulsion. For the concerted mechanism (Figure 1B) there are two common regions of non-covalent interactions: (1) C-H van-der-Waals interactions of sugar backbone with catalyst adamantyl group and (2) enol ether $\mathrm{C}-\mathrm{H}$ interaction with $\pi$-system of catalyst 3-aryl system. The most important interaction (3) is between the 4,6-benzylidene group and CPA isopropyl groups (circled for $\mathbf{T S}_{\mathbf{2}}$ ). The presence of such interaction can destabilize the transition state and make the C3-protection less favorable than the corresponding C2-pathway that lacks such steric repulsion (3). Remarkably, during the C2protection via the phosphate-mediated mechanism, reactants and catalyst in $\mathbf{T S}_{\mathbf{4}}$ are aligned almost in the same ways as for the concerted reaction (TS $\mathbf{T S}_{1}$. There are some weak $\pi \cdots \mathrm{H}-\mathrm{C}$ interactions (4) that appear between the substrate $\mathbf{4 a}$ and the MOC protecting group PG, but interactions (1) and (2) with the catalyst skeleton also are present. These similarities in NCI patterns for the $\mathbf{T S}_{\mathbf{1}}$ and $\mathbf{T S}_{\mathbf{4}}$ are in good agreement with almost identical TS energies for the C2-acetalyzation for both mechanisms. In contrast, the molecular alignment in $\mathbf{T S}_{\mathbf{5}}$ (C3-protection) is significantly different from the concerted mechanism. The reactants are aligned in a more compact way and positioned further away from the catalytic pocket. The NCIs (1) and (2) arising from the 2,3-diol 4a, MOC enol ether PG and the catalyst are minimized, and only interaction (4) between the reactants are clearly present. The absence of these NCIs in $\mathbf{T S}_{\mathbf{5}}$ leads to its higher stability and, thus, the C3-protection prevails for the phosphate-mediated mechanism. Identified interactions and reactants alignment in catalytic cavity can be extrapolated and proved by looking at other substrates. First, using less bulky acetonide-protected compound $\mathbf{4 m}$ gives only 12:1 regioselectivity favoring the $\mathrm{C} 2$-protected product. Two methyl groups in transition state $\mathbf{T S}_{\mathbf{2}}-\mathrm{C} 3$ should cause fewer destabilizing interactions with isopropyl groups reducing selectivity. Bulky C1-susbtituents in $\beta$-configuration $\left(-\mathrm{SC}_{6} \mathrm{H}_{4} t \mathrm{Bu}\right.$ and $\left.-\mathrm{SNapth}\right)$ shouldn't impact selectivity as they are positioned far away from catalyst and it is also in agreement with experimental data (substrates $\mathbf{4 b}$ and $\mathbf{4 d}$ also gives high 25:1 selectivity). Transition state $\mathbf{T S}_{\mathbf{1}}-\mathrm{C} 2$ reveals potential interactions of catalyst backbone with $\mathrm{C1}$ susbtituent in $\alpha$-configuration leading to its destabilization and to C3-selectivity as it was observed in case with $D$-glucose derivative $\mathbf{4 f}$.

In summary, the concerted asynchronous (Path B) and phosphate intermediate (Path $\mathrm{C}$ ) mechanisms feature similar interactions in the transition states and molecular positions for the formation of the $\mathrm{C} 2$ isomer, which are reflected in negligible energy differences between these two pathways. Thus, the regioselectivity for the CPA-catalyzed acetal formation is determined by the steric interactions in the TS for the C3-protection. At low temperatures these results are consistent with the concerted mechanism being operative, where the sugar substrate alignment leading to the C3 product suffers from the interactions of 4,6-benzylidene acetal moiety with the catalyst. Considering the small difference in the RDS energy barriers for both pathways, the temperature can be an important factor in favoring one mechanism-and therefore one regioselectivity-over another.

\section{CONCLUSION}

In conclusion, this article provides full account on regioselective acetal protection of monosaccharide-based diols using chiral phosphoric acid $\mathbf{2}$ as the catalyst. These catalystcontrolled regioselective acetalizations were found to proceed with high regioselectivities (up to $>25: 1 \mathrm{rr}$ ) on various $D$-glucose, $D$-galactose, $D$-mannose and $L$-fucose derived 1,2-diols and could be carried with immobilized and recyclable catalyst $(\boldsymbol{R})$-Ad-TRIP-PS (3). The utility of regioselective CPA-catalyzed acetalizations was further demonstrated for the regioselective single-pot preparation of various differentially protected mono- and disaccharide building blocks in good yields. The computational and mechanistic studies indicate complex temperature-dependent interplay of two reaction mechanisms. The dominant at low temperatures concerted asynchronous mechanism favors the formation of the $\mathrm{C} 2$ isomer due to destabilizing the TS leading to the $\mathrm{C} 3$ isomer interactions between the benzylidene acetal moiety of $\mathbf{4 a}$ and catalyst 2 . The developed stereochemical models allow expanding the scope of this transformation, and further studies focused on exploring more complex substrates and applying these transformations in continuous flow are the subjects of ongoing studies by our groups.

\section{ASSOCIATED CONTENT}

\section{Supporting Information}

Experimental procedures, ${ }^{1} \mathrm{H}$ and ${ }^{13} \mathrm{C}$ NMR spectra, and more detailed description of computational studies, are available free of charge via the Internet.

\section{AUTHOR INFORMATION}

\section{Corresponding Authors}

* nagorny@umich.edu, *paulzim@umich.edu

\section{Author Contributions}

§J.L. and S.W. are equally-contributing authors. All authors have given approval to the final version of the manuscript.

\section{Funding Sources}

PN and PZ thank NIH Common Fund grant U01GM125274 for supporting this work. 


\section{Acknowledgement}

We thank Prof. John Montgomery for the useful discussions during the preparation of this manuscript.

\section{REFERENCES}

(1) (a) Ernst, B; Magnani, J. L. "From carbohydrate leads to glycomimetic drugs" Nature Rev. Drug Disc. 2009, 8, 661; (b) Lepenies, B.; Seeberger, P. H. "The promise of glycomics, glycan arrays and carbohydrate-based vaccines" Immunoph. Immunotoxic. 2010, 32, 196.

(2) (a) C. S. Bennett "Principles of modern solid-phase oligosaccharide synthesis" Org. Biomol. Chem. 2014, 12, 1686; (b) Smoot, J. T.; Demchenko, A. V. "Oligosaccharide synthesis: from conventional methods to modern expeditious strategies" Ad. Carbohydr. Chem. Biochem. 2009, 62, 161; (c) Krasnova, L.; Wong, C.-H., "Oligosaccharide synthesis and translational innovation"J. Am. Chem. Soc. 2019, 141, 3735; (d) Seeberger, P. H.; Werz, D. B. "Synthesis and medical applications of oligosaccharides" Nature 2007, 446, 1046; (e) Seeberger, P. H.; Haase, W.-C. "Solid-phase oligosaccharide synthesis and combinatorial carbohydrate libraries" Chem. Rev. 2000, 100,4349 .

(3) (a) Filice, M.; Palomo, J. M. "Monosaccharide derivatives as central scaffolds in the synthesis of glycosylated drugs" RSC Advances 2012, 2, 1729; (b) Wang, T.; Demchenko, A. V. "Synthesis of carbohydrate building blocks via regioselective uniform protection/deprotection strategies" Org. Biomol. Chem. 2019, 17, 4934; (c) Zulueta, M. M. L.; Janreddy, D.; Hung, S.-C. "One-pot methods for the protection and assembly of sugars" Isr. J. Chem. 2015, 55, 347.

(4) (a) Wang, C.-C.; Lee, J.-C.; Luo, S.-Y.; Kulkarni, S. S.; Huang, Y.-W.; Lee, C.-C.; Chang, K.-L.; Hung, S.-C. "Regioselective one-pot protection of carbohydrates" Nature 2007, 446, 896; (b) Wang, C.-C.; Kulkarni, S. S.; Lee, J.-C.; Luo, S.-Y.; Hung, S.-C. "Regioselective one-pot protection of glucose" Nature Prot. 2008, 3, 98; (c) Huang, T.-Y.; Zulueta, M. M. L.; Hung, S.-C. "Regioselective one-pot protection, protection-glycosylation and protection-glycosylation-glycosylation of carbohydrates: a case study with D-glucose" Org. Biomol. Chem. 2014, 12, 376

(5) (a) Francais, A.; Urban, D.; Beau, J.-M. "Tandem catalysis for a one-pot regioselective protection of carbohydrates: the example of glucose" Angew. Chem. Int. Ed. 2007, 46, 8662; (b) Beau, J.-M.; Bourdreux, Y.; Despras, G.; Gouasmat, A.; San Jose, G.; Urban, D.; Vauzeilles, B. "One-pot multistep regioselective protection of carbohydrates catalyzed by acids" Protecting Groups: Strategies and Applications in Carbohydrate Chemistry, First Edition, 2019, Wiley-VCH Verlag GmbH \& Co.

(6) (a) Giuliano, M. W.; Miller, S. J. "Site-selective reactions with peptide-based catalysts" Top. Curr. Chem. 2016, 372, 157; (b) Ueda, Y.; Kawabata, T. "Organocatalytic site-selective acylation of carbohydrates and polyol compounds" Top. Curr. Chem. 2016, 372, 303; (c) Lee, D.; Taylor, M. S. "Catalyst-controlled regioselective reactions of carbohydrate derivatives" Synthesis 2012, 44, 3421; (d) Lawandi, J.; Rocheleau, S.; Moitessier, N. "Regioselective acylation, alkylation, silylation and glycosylation of monosaccharides" Tetrahedron 2016, 72, 6283; (e) Dimakos, V.; Taylor, M. S. "Site-selective functionalization of hydroxyl groups in carbohydrate derivatives" Chem. Rev. 2018, 118, 11457.

(7) Selected examples of site-selective achiral catalyst promoted protection of sugars: (a) Dimakos, V.; Garrett, G. E.; Taylor, M. S. "Site-selective, copper-mediated 0-arylation of carbohydrate derivatives" J. Am. Chem. Soc. 2017, 139, 15515; (b) Lee, D.; Taylor, M. S. "Borinic acid-catalyzed regioselective acylation of carbohydrate derivatives" J. Am. Chem. Soc. 2011, 133, 3724; (c) Wu, J.; Li, X.; Qi, X.; Duan, X.; Cracraft, W. L.; Guzei, I. A.; Liu, P.; Tang, W. "Site-selective and stereoselective 0-alkylation of glycosides by Rh(II)catalyzed carbenoid insertion"J. Am. Chem. Soc. 2019, 141, 19902; (d) Peng, P.; Linseis, M.; Winter, R. F.; Schmidt, R. R. "Regioselective acylation of diols and triols: the cyanide effect" J. Am. Chem. Soc. 2016, 138, 6002; (e) Li, T.; Li, T.; Linseis, M.; Wang, F.; Winter, R. F.; Schmidt, R. R.; Peng, P. "Catalytic regioselective benzoylation of 1,2-trans-diols in carbohydrates with benzoyl cyanide: the axial oxy group effect and the action of achiral and chiral amine catalysts" ACS Catalysis 2020, 10, 11406; (f) Huber, F.; Kirsch, S. F. “Siteselective acylations with tailor-made catalysts" Chem.-Eur. J. 2016 22, 5914.

(8) (a) Sculimbrene, B. R.; Miller, S. J. "Discovery of a catalytic asymmetric phosphorylation through selection of a minimal kinase mimic: a concise total synthesis of D-myo-inositol-1-phosphate" J. Am. Chem. Soc. 2001, 123, 10125; (b) Sculimbrene, B. R.; Morgan, A. J.; Miller, S. J. "Enatniodivergence in small-molecule catalysis of asymmetric phosphorylation: concise total syntheses of the enantiomeric D-myo-inositol-1-phosphate and D-myo-inositol-3-phosphate" J. Am. Chem. Soc. 2002, 124, 11653; (c) Griswold, K. S.; Miller, S. J. "A peptide-based catalyst approach to regioselective functionalization of carbohydrates" Tetrahedron 2003, 59, 8869; (d) Allen, C. L.; Miller, S. "Chiral copper(II) complex-catalyzed reactions of partially protected carbohydrates" J. Org. Lett. 2013, 15, 6178.

(9) (a) Kawabata, T.; Muramatsu, W.; Nishio, T.; Shibata, T.; Schedel, $\mathrm{H}$. "A catalytic one-step process for the chemo- and regioselective acylation of monosaccharides" J. Am. Chem. Soc. 2007, 129, 12890; (b) Muramatsu, W.; Mishiro, K.; Ueda, Y.; Furuta, T.; Kawabata, T. "Perfectly regioselective and sequential protection of glucopyranosides" Eur. J. Org. Chem. 2010, 5, 827.

(10) (a) Xiao, G.; Cintron-Rosado, G. A.; Glazier, D. A.; Xi, B.; Liu, C.; Tang, W. "Catalytic site-selective acylation of carbohydrates directed by cation-n interactions" J. Am. Chem. Soc. 2017, 139, 4346; (b) Blaszczyk, S. A.; Xiao, G.; Wen, P.; Hao, H.; Wu, J.; Wang, B.; Carattino, F.; Li, Z.; Glazier, D. A.; McCarty, B. J.; Liu, P.; Tang, W. "S-adamantyl group directed site-selective acylation and its applications in the streamlined assembly of oligosaccharides" Angew. Chem. Int. Ed. 2019, 58, 9542.

(11) Selected publications on chiral catalyst-controlled functionalization of carbohydrates: (a) Li, R.-Z.; Tang, H.; Wan, L.; Zhang, X.; Fu, Z.; Liu, J.; Yang, S.; Jia, D.; Niu, D. "Site-divergent delivery of terminal propargyls to carbohydrates by synergistic catalysis" Chem 2017, 3, 834; (b) Sun, X.; Lee, H.; Lee, S.; Tan, K. L. "Catalyst recognition of cis-1,2-diols enables site-selective functionalization of complex molecules" Nature Chem. 2013, 5, 790; (c) Shang, W.; Mou, Z.-D.; Tang, H.; Zhang, X.; Liu, J.; Fu, Z.; Niu, D. "Site-selective O-arylation of glycosides" Angew. Chem. Int. Ed. 2018, 57, 314; (d) Hu, G.; Vasella, A. "Regioselective benzoylation of 6-0-protected and 4,60 -diprotected hexopyranosides as promoted by chiral and achiral ditertiary 1,2-diamines" Helv. Chim. Acta 2002, 85, 4369.

(12) (a) Lee, J.; Borovika, A.; Khomutnyk, Y.; Nagorny, P. “ Chiral phosphoric acid-catalyzed desymmetrizative glycosylation of 2 deoxystreptamine and its application to aminoglycoside synthesis" Chem. Commun. 2017, 53, 8976; b) Tay, J.-H.; Arguelles, A. J.; DeMars II, M. D.; Zimmerman, P. M.; Sherman, D. H.; Nagorny, P." Chiral Phosphoric Acid-controlled Regioselective Glycosylation of 12membered Macrolides" J. Am. Chem. Soc. 2017, 139, 8570; c) Khomutnyk, Y. Y.; Arguelles, A. J.; Winschel, G. A.; Sun, Z.; Zimmerman, P. M.; Nagorny, P. “ Studies of the Mechanism and Origins 
of Enantioselectivity for the Chiral Phosphoric Acid-Catalyzed Stereoselective Spiroketalization" J. Am. Chem. Soc. 2016, 138, 144; d) e) Nagorny, P.; Sun, Z.; Winschel, G. A. "Chiral Phosphoric Acid Catalyzed Stereoselective spiroketalizations" Synlett 2013, 24, 661; f) Sun, Z.; Winschel, G. A.; Borovika, A.; Nagorny, P. "Chiral Phosphoric Acid-Catalyzed Enantioselective and Diastereoselective Spiroketalizations Chiral Phosphoric Acid-Catalyzed Enantioselective and Diastereoselective Spiroketalizations" J. Am. Chem. Soc. 2012, 134,8074

(13) Mensah, E.; Camasso, N.; Kaplan, W.; Nagorny, P. “ Chiral Phosphoric Acid-Directed Regioselective Acetalization of Carbohydrate-Derived 1,2-diols" Angew. Chem. Int. Ed. 2013, 52, 13939.

(14) Selected examples of CPA-catalyzed stereoselective formation and cleavage of acetal protecting groups: (a) Kim, J. H.; Coric, I.; Palumbo, C.; List, B. "Resolution of diols via catalytic asymmetric acetalization" J. Am. Chem. Soc. 2015, 137, 1778; (b) Yamanaka, T.; Kondoh, A.; Terada, M. "Kinetic resolution of racemic amino alcohols through intermolecular acetalization catalyzed by a chiral Bronsted acid" J. Am. Chem. Soc. 2015, 137, 1048; (c) Ye, B.; Zhao, J.; Zhao, K.; McKenna, J. M.; Toste, D. F. “Chiral diaryliodonium phosphate enables light driven diastereoselective a-C(sp3)-H acetalization" J. Am. Chem. Soc. 2018, 140, 8350; (d) Meng, S.-S.; Liang, Y.; Cao, K.-S.; Zou, L.; Lin, X.-B.; Yang, H.; Houk, K. N.; Zheng, W.-H. “Chiral phosphoric acid catalyzed highly enantioselective desymmetrization of 2-substituted and 2,2-disubstituted 1,3-diols via oxidative cleavage of benzylidene acetals" J. Am. Chem. Soc. 2014, 136, 12249; (e) Hyudo, K.; Gandhi, S.; van Gemmeren, M.; List, B. "Bronsted acid catalyzed asymmetric silylation of alcohols" Synlett 2015, 26, 1093; (f) Kimura, T.; Sekine, M.; Takahashi, D.; Toshima, K. "Chiral Bronsted acid mediated glycosylation with recognition of alcohol chirality" Angew. Chem. Int. Ed. 2013, 52, 12131.

(15) (a) Akiyama, T.; Itoh, J.; Yokota, K.; Fuchibe, K. "Enantioselective Mannich-type reaction catalyzed by a chiral Bronsted acid" Angew. Chem. Int. Ed. 2004, 43, 1566; (b) Uraguchi, D.; Terada, M. "Chiral Bronsted acid-catalyzed direct Mannich reactions via electrophilic activation" J. Am. Chem. Soc. 2004, 126, 5356.

(16) Hayashi, Y. "Pot economy and one-pot synthesis" Chem. Science 2016, 7, 866.

(17) (a) Wuts, P. G. M.; Greene, T. W. Green's Protective Groups in Organic Synthesis, $4^{\text {th }}$ ed.; John Wiley \& Sons, Inc.; Hoboken, NJ. 2007, pp 59-79; b) Kocienski, P. J. Protecting Groups, 3d ed.; Georg Thieme Verlag; Stuttgart 2005, pp 315-320.

(18) (a) Benaglia, M.; Puglisi, A.; Cozzi, F. "Polymer-supported organic catalysts" Chem. Rev. 2003, 103, 3401; (b) Kristensen, T. E.; Hansen, T. "Polymer-supported chiral organocatalysts: synthetic strategies for the road towards affordable polymeric immobilization" Eur. J. Org. Chem. 2010, 17, 3179; (c) Rodriguez-Escrich, C.; Pericas, M. A. "Organocatalysis on tap: enantioselective continuous flow processes mediated by solid-supported chiral organocatalysts" Eur. J. Org. Chem. 2015, 6, 1173; (d) Rodriguez-Escrich, C.; Pericas, M. A. "Catalytic enantioselective flow processes with solidsupported chiral catalysts" Chem. Rec. 2019, 19, 1872.

(19) (a) Bartoszek, M.; Beller, M.; Deutsch, J.; Klawonn, M.; Kockritz, A.; Nemati, N.; Pews-Davtyan, A. "A convenient protocol for the synthesis of axially chiral Brønsted acids" Tetrahedron 2008, 64, 1316; (b) Rueping, M.; Sugiono, E.; Steck, A.; Theissmann, T. "Synthesis and application of polymer-supported chiral Brønsted acid organocatalysts" Adv. Synth. Catal. 2010, 352, 281; (c) Bleschke, C.; Schmidt, J.; Kundu, D. S.; Blechert, S.; Thomas, A. "A chiral microporous polymer network as asymmetric heterogeneous organocatalyst" Adv. Synth. Catal. 2011, 353, 3101; (d) Kundu, D. S.; Schmidt, J.; Bleschke, C.; Thomas, A.; Blechert, S. "A microporous
BINOL-derived phosphoric acid" Angew. Chem. Int. Ed. 2012, 51, 5456; (e) Osorio-Planes, L.; Rodriguez-Escrih, C.; Pericas, M. A. “Enantioselective continuous-flow production of 3-indolylmethanamines mediated by an immobilized phosphoric acid catalyst" Chem. Eur. J. 2014, 20, 2367.

(20) Clot-Almenara, L.; Rodriguez-Escrich, C.; Osorio-Planes, L.; Pericas, M. A. "Polystyrene-supported TRIP: a highly recyclable catalyst for batch and flow enantioselective allylation of aldehydes" ACS Catal. 2016, 6, 7642 .

(21) (a) Kanomata, K.; Toda, Y.; Shibata, Y.; Yamanaka, M.; Tsuzuki, S.; Gridnev, I. D.; Terada, M. "Secondary stereocontrolling interactions in chiral Bronsted acid catalysis: study of a Petasis-Ferriertype rearrangement catalyzed by chiral phosphoric acids" Chem. Sci. 2014, 5, 3515; (b) Maskeri, M. A.; Brueckner, A. C.; Feoktistova, T.; O'Connor, M. J.; Walden, D. M.; Cheong, P. H.-Y.; Scheidt, K. A. "Mechanism and origins of selectivity in the enantioselective oxaPicter-Spengler reaction: A cooperative catalytic complex from a hydrogen bond donor and chiral phosphoric acid" Chem. Sci. 2020, 11, 8736; (c) Lee, S.; Kaib, P. S. J.; List, B. "Asymmetric catalysis via cyclic, aliphatic oxocarbenium ion" J. Am. Chem. Soc. 2017, 139, 2156; (d) Das, S.; Liu, L.; Zheng, Y.; Alachraf, M. W.; Thiel, W.; De, C. K.; List, B. "Nitrated confined imidodiphosphates enable a catalytic asymmetric oxa-Pictet-Spengler reaction" J. Am. Chem. Soc. 2016, 138,9429

(22) (a) Adero, P. O.; Amarasekara, H.; Wen, P.; Bohe, L.; Crich, D. "The experimental evidence in support of glycosylation mechanisms at the SN1-SN2 interface" Chem. Rev. 2018, 118, 8242; (b) $\mathrm{Fu}$, Y.; Bernasconi, L.; Liu, P.; "Ab initio molecular dynamics simulations of the SN1/SN2 mechanistic continuum in glycosylation reactions"J. Am. Chem. Soc. 2021, 143, 1577; (c) Varner, T. P.; Teator, A. J.; Reddi, Y.; Jacky, P. E.; Cramer, C. J.; Leibfarth, F. A. "Mechanistic insights into the stereoselective cationic polymerization of vinyl ethers" J. Am. Chem. Soc. 2020, 142, 17175.

(23) Sun, Z.; Winschel, G. A.; Zimmerman, P. M.; Nagorny, P. “Enantioselective synthesis of piperidines through the formation of mixed chiral phosphoric acid acetals: experimental and theoretical studies" Angew. Chem. Int. Ed. 2014, 53, 11194.

(24) Zimmerman, P. "Reliable Transition State Searches Integrated with the Growing String Method" J. Chem. Theory Comput. 2013, 9, 3043.

(25) Zimmerman, P. M. "Single-Ended Transition State Finding with the Growing String Method" J. Comput. Chem. 2015, 36, 601.

(26) Contreras-García, J.; Johnson, E. R.; Keinan, S.; Chaudret, R.; Piquemal, J.; Beratan, D. N.; Yang, W. "NCIPLOT: A Program for Plotting Noncovalent Interaction Regions" J. Chem. Theory Comput. 2011, 7, 625 . 


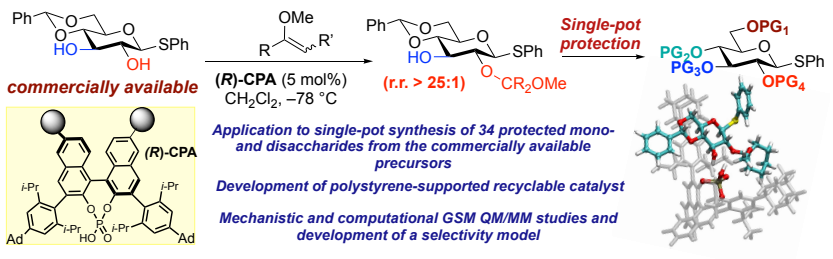

